\title{
Heat Shock Protein A12B Protects Vascular Endothelial Cells Against Sepsis-Induced Acute Lung Injury in Mice
}

\author{
Yi Chen ${ }^{\mathrm{a}}$ Lei Wang ${ }^{\mathrm{a}}$ Qiuxiang Kang ${ }^{\mathrm{a}}$ Xu Zhang ${ }^{\mathrm{a}}$ Guifang Yu ${ }^{\mathrm{b}}$ Xiaojian Wan ${ }^{\mathrm{a}}$

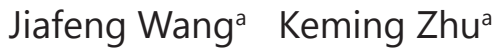 \\ aDepartment of Anesthesiology and Intensive Care Medicine, Changhai Hospital, the Second Military \\ Medical University, bepartment of Anesthesiology, the Third People's Hospital, Shanghai Jiaotong \\ University, Shanghai, China
}

\section{Key Words}

Sepsis • Acute lung injury • Vascular endothelial cells $•$ Heat shock protein A12B

\begin{abstract}
Background: Pulmonary endothelial injury is a critical process in the pathogenesis of acute lung injury (ALI) during sepsis. Heat shock protein A12B (HSPA12B) is mainly expressed in endothelial cells and protects against several harmful factors. However, the effects of HSPA12B in sepsis-induced ALI and its potential mechanisms of action remain unclear. Methods: For in vivo experiments, C57BL/6 mice were randomly divided into four groups $(n=15)$ : a sham operation group, a cecal ligation and puncture (CLP) group, a HSPA12B siRNA-CLP group and a negative control (NC) siRNA-CLP group. The mice were treated by nasal inhalation of 2-OMemodified HSPA12B siRNA or NC siRNA. Sepsis was induced by CLP. Samples were harvested 24 and 48 hours post-CLP surgery. Pathological changes and scoring of lung tissue samples were monitored using hematoxylin and eosin staining. Levels of pro-inflammatory cytokines (e.g., interleukin (IL)-1 $\beta$, tumor necrosis factor (TNF)- $\alpha$, and IL-6) and myeloperoxidase activity in bronchoalveolar lavage fluid were analyzed by ELISA. Pulmonary edema was assessed using a wet-to-dry weight ratio. Neutrophils and alveolar macrophages were counted using flow cytometry. Pulmonary endothelial cell apoptosis was detected by TUNEL staining. Expression levels of MAPK family signaling molecules and caspase- 3 were measured by Western blot analysis. In addition, 7-day survival was recorded. For in vitro experiments, human umbilical vein endothelial cells were pre-transfected with HSPA12B siRNA or PIRES2-EGFP-HSPA12BFlag plasmid and treated with lipopolysaccharide; subsequently, the expression levels of MAPK family signaling molecules and caspase- 3 were measured by Western blotting. Results: Nasal inhalation of nano-polymer-encapsulated HSPA12B siRNA specifically downregulated mRNA and protein expression levels of HSPA12B in lung tissues. The administration of HSPA12B siRNA aggravated lung pathological injury, upregulated pro-inflammatory cytokine (e.g., IL$1 \beta$, TNF- $\alpha$, and IL-6) expression, and increased myeloperoxidase activity, neutrophil infiltration, pulmonary edema, and pulmonary endothelial cell apoptosis. Additionally, HSPA12B Y. Chen and L. Wang contributed equally to this work.
\end{abstract}


knockdown worsened survival after CLP surgery. The potential protective mechanisms of HSPA12B may involve the inhibition of ERK phosphorylation and caspase- 3 activation in vivo and in vitro. Conclusion: HSPA12B protected against sepsis-induced ALI. The potential mechanism may be partly due to the inhibition of ERK phosphorylation and caspase- 3 activation. These findings provide a potential therapeutic target for treating sepsis.

(C) 2017 The Author(s)

Published by S. Karger AG, Basel

\section{Introduction}

Sepsis is a systemic inflammatory response that affects several organs [1]. The death of patients with severe sepsis is primarily caused by multiple organ dysfunction. The respiratory system is the most frequently affected organ system $[2,3]$, and nearly $50 \%$ of patients with severe sepsis will develop acute lung injury (ALI) in its more severe form, known as acute respiratory distress syndrome (ARDS) [4].

ALI is involved in several key pathological processes, including loss of vascular integrity, neutrophil infiltration, and the accumulation of protein-rich fluid in the airspaces of the lungs. Pulmonary endothelial injury is critical to the pathogenesis of ALI during sepsis [5-7]. Endothelial cells attach to the lining of vessel walls and regulate blood flow, thus facilitating the delivery of cells and proteins to tissue. Difficulties in healing from endothelial cell injury can lead to the destruction of the blood-air barrier, which directly results in the leakage of proteins from lung tissue, triggering ALI and potentially ARDS [8]. Thus, identifying genes that modulate sepsis-induced endothelial dysfunction could contribute to future therapeutic interventions for sepsis-induced ALI/ARDS.

Heat shock protein A12B (HSPA12B) is a member of a subfamily of proteins that is distantly related to the heat shock protein 70 family. HSPA12B is mainly expressed in endothelial cells and is also widely distributed in various tissues in mammals [9]. Many studies have found that HSPA12B protects endothelial cells against a variety of harmful factors, such as cerebral ischemia/reperfusion injury and cardiac dysfunction [10-13]. Moreover, HSPA12B can reduce lipopolysaccharide (LPS)-induced endothelial injury in human umbilical vein endothelial cells (HUVECs) via the PI3K/Akt pathway [14]. In addition, we previously found that HSPA12B protects against sepsis-induced impairment in vascular endothelial (VE) permeability via VE-cadherin upregulation [15]. Furthermore, a clinical trial showed not only that the plasma level of HSPA12B was elevated in both septic mice and patients but also that it might be a good prognostic predictor in patients with sepsis [16]. However, the effect of HSPA12B on sepsis-induced pulmonary endothelial injury and its potential mechanisms of action have remained unclear.

To investigate the function and potential mechanism of HSPA12B action in sepsismediated ALI, we treated mice with a nasal inhalation of pre-designed HSPA12B siRNA packaged in the non-liposomal material known as Entranster ${ }^{\mathrm{TM}}$-in vivo. We assessed transfection efficiency, and after performing cecal ligation and puncture (CLP) to artificially induce sepsis, the mice were monitored for survival and potential lung injury. Here, we identify HSPA12B as a novel molecule capable of regulating vascular endothelial function during sepsis-induced lung injury.

\section{Materials and Methods}

Chemicals and Reagents

Entranster ${ }^{\mathrm{TM}}$-in vivo was purchased from Engreen Biosystem Ltd. (China). siRNAs were designed and synthesized in 2'-OMe-modified form by GenePharma Research (China) (Table 1). Escherichia coli LPS was purchased from Sigma-Aldrich (St. Louis, MO, USA); ELISA kits for mouse interleukin (IL)-1 $\beta$, tumor necrosis factor (TNF)- $\alpha$, and IL-6 were purchased from R\&D Systems Inc. (Minneapolis, MN). Myeloperoxidase (MPO) activity was measured with a mouse MPO kit obtained from Abcam (Cambridge, MA). RIPA buffer 


\section{Cellular Physiology Cell Physiol Biochem 2017;42:156-168 \begin{tabular}{l|l} 
and Biochemistry Published onIIne: May 18, 2017 & $\begin{array}{l}\text { C } 2017 \text { The Author(s). Published by S. Karger AG, Basel } \\
\text { www.karger.com/cpb }\end{array}$
\end{tabular} \\ Chen et al.: HSPA12B Protects Vascular Endothelium in Sepsis-Induced ALI}

Table 1. siRNA sequences for animal treatment. Forward (F) and reverse (R) primers were designed for each gene as specified below

\begin{tabular}{|c|c|c|}
\hline Gene & Forward primer & Reverse primer \\
\hline Negative Control & 5'-UUCUCCGAACGUGUCACGUTT-3' & 5'-ACGUGACACGUUCGGAGAATT-3' \\
\hline HSPA12B & 5'-GGGCUGUAUAUCAGCUCUATT-3' & 5'-UAGAGCUGAUAUACAGCCCTT-3' \\
\hline
\end{tabular}

Table 2. Primer sequences for RT-qPCR. Forward (F) and reverse (R) primers were designed for each gene as specified below

\begin{tabular}{|c|c|c|}
\hline Gene & Forward primer & Reverse primer \\
\hline$\beta$-Actin & 5'-TGAGAGGGAAATCGTGCGTG-3' & 5'-TTGCTGATCCACATCTGCTGG-3' \\
\hline HSPA12B & 5'-CCCATGCCCTGCGCTTCTTC-3' & 5'-ACTGCTTGGCTGGCTGTTTC-3' \\
\hline
\end{tabular}

was obtained from Cell Signaling Technology (Beverly, MA, USA). Anti-HSPA12B antibody was purchased from Abnova (Taipei, Taiwan); a MAPK family antibody sample kit and goat anti-rabbit IgG-HRP secondary antibody were obtained from Cell Signaling Technology (Beverly, MA, USA); anti-GAPDH antibody was purchased from R\&D Systems Inc. (Minneapolis, MN, USA); and anti-caspase-3 antibody was purchased from Abcam (Cambridge, MA).

\section{Animals}

Male C57BL/6J mice were housed in a laboratory animal facility under a 12-hour light-dark cycle and were given free access to water and normal chow. Mice aged 6 to 8 weeks and ranging from 20 to $25 \mathrm{~g}$ in weight were used for subsequent experiments. The mice were purchased from the Second Military Medicine University Animal Center (Shanghai, China). All procedures were approved by the Animal Care and Use Committee of Changhai Hospital (Shanghai, China) in compliance with the National Institutes of Health Guidelines.

\section{Delivery of Entranster ${ }^{T M}$-In Vivo/siRNA Complex}

Mice were anesthetized via inhalation of sevoflurane and then intranasally administered HSPA12B siRNA ( $2 \mathrm{mg} / \mathrm{kg}$ of body weight) or an equivalent dose of negative control (NC) siRNA. The siRNA was diluted with diethylpyrocarbonate to a concentration of $1 \mu \mathrm{g} / \mu \mathrm{l}$ based on optical density (OD), thus constituting a nucleic acid $(\mu \mathrm{g})$-to-Entranster ${ }^{\mathrm{TM}}$-in vivo $(\mu \mathrm{l})$ ratio of 1:0.5. The solution was thoroughly mixed and allowed to stabilize at room temperature for 15 minutes. The duration of inhalation was maintained at 10 minutes to facilitate the complete absorption of the siRNA mixture into the nasal mucosa.

\section{CLP Model}

CLP was performed on C57BL/6J mice $(n=15)$ as previously described [17]. After the mice were anaesthetized via inhalation of $2 \%$ sevoflurane (Baxter, USA), the cecum was exposed through a $1.5-\mathrm{cm}$ incision in the lower quadrants of the abdomen. Then, the distal three-fourths of the cecum was ligated with 4-0 silk suture and subsequently punctured through with a 22-gauge needle. The cecum was repositioned, and the abdominal incision was closed by two-layer suturing. After surgery, the mice were injected subcutaneously with $1 \mathrm{ml}$ of sterile saline and provided free access to food and water after awakening. No antibiotics were administered to the mice after CLP, as we performed CLP to artificially induce sepsis and assess the effect of HSPA12B siRNA on sepsis-mediated ALI.

\section{Real-time Quantitative Polymerase Chain Reaction (RT-qPCR)}

Total RNA was prepared from lung tissues and HUVECs using TRIzol reagent (Invitrogen Corp., Carlsbad, CA) according to the manufacturer's instructions. The primers used for PCR were designed to be isoform-specific (Table 2). Following the RT-qPCR kit instructions, $500 \mathrm{ng}$ of purified total RNA was used to obtain cDNA through reverse transcription. Gene transcripts were quantified with a SYBR Premix Ex Taq Kit (Takara, Kyoto, Japan). A quantitative PCR system was prepared with $10 \mu \mathrm{l}$ of SYBR Premix, $1 \mu \mathrm{l}$ of forward and reverse primers at $10 \mu \mathrm{mol} / \mathrm{l}$, and $1 \mu \mathrm{l}$ of cDNA and deionized water, for a total reaction volume of $20 \mu \mathrm{l}$. The detection conditions were as follows: $95^{\circ} \mathrm{C}$ for $30 \mathrm{sec}$, followed by 40 cycles of $5 \mathrm{sec}$ at $95^{\circ} \mathrm{C}$ and $30 \mathrm{sec}$ 


\section{Cellular Physiology Cell Physiol Biochem 2017;42:156-168 \begin{tabular}{l|l} 
DOI: 10.1159/000477308 & $\begin{array}{l}\text { O 2017 The Author(s). Published by S. Karger AG, Basel } \\
\text { www.karger.com/cpb }\end{array}$
\end{tabular} \\ Chen et al.: HSPA12B Protects Vascular Endothelium in Sepsis-Induced ALI}

at $60^{\circ} \mathrm{C}$. All the data were analyzed using $\beta$-actin as an internal control. The relative copies of the target gene were determined using the $2^{\text {-DDCt }}$ method

\section{Western Blot Analysis}

Lung tissues and HUVECs were collected, and protein was extracted. Protein concentrations were determined using a BCA protein assay kit (Pierce, USA). Total protein was separated by $10 \%$ SDS-PAGE and then transferred to PVDF membranes. The membranes were incubated for $1 \mathrm{~h} \mathrm{in} 5 \%$ non-fat milk and Tris-buffered saline with Tween (TBST; 0.01\% Tween 20, $20 \mathrm{mM}$ Tris, pH 7.6, and $137 \mathrm{mM} \mathrm{NaCl}$ ), which was followed by an overnight incubation at $4{ }^{\circ} \mathrm{C}$ with the following primary antibodies: anti-GAPDH monoclonal antibody (diluted 1:2000), anti-HSPA12B monoclonal antibody, MAPK family antibody, and anti-caspase-3 antibody (diluted 1:1000). On the following day, the membranes were washed with TBST three times. The membranes were then incubated for $2 \mathrm{~h}$ at room temperature with donkey anti-rabbit or mouse antirabbit secondary antibody (diluted 1:2000) and subsequently washed with TBST three times. Proteins were visualized with an enhanced chemiluminescence kit (Pierce, USA). The density of protein bands was quantified by ImageJ software using GAPDH as a reference.

\section{Bronchoalveolar lavage fluid (BALF)}

Both lungs of each mouse were lavaged three times through a tracheal cannula with $0.5 \mathrm{ml}$ of phosphatebuffered saline (PBS) at $4^{\circ} \mathrm{C}$, instilled up to a total volume of $1 \mathrm{ml}$. Then, the BALF was centrifuged $(3,000$ $\mathrm{rpm}, 10 \mathrm{~min}, 4^{\circ} \mathrm{C}$ ). The supernatant was harvested for ELISA according to the manufacturer's instructions. The centrifuged cells were then prepared for a flow cytometric analysis of neutrophil (anti-mouse Ly-6GFITC, eBioscience) and alveolar macrophage (anti-mouse F4/80 antigen-APC, eBioscience) count. The protein content of the BAL supernatant was determined using a BCA protein assay kit.

\section{Histopathological Lung Examination}

The right lungs were dissected, fixed in $4 \%$ paraformaldehyde, embedded in paraffin, and sectioned at $5 \mu \mathrm{m}$ for hematoxylin and eosin (H\&E) staining and histological examination. Pathology scores were graded using the Carraway double-blind method [18] by two independent pathology specialists; scores were based on either pulmonary interstitial thickening, alveolar edema, alveolar hemorrhage, and neutrophil infiltration (normal, 0 ; mild, 1; medium, 2; severe, 3) or the extent of disease (normal, $0 ;<25 \%, 1 ; 25-50 \%$, $2 ;>50 \%, 3$ ). In each section from each group at each time point, two fields of view were selected from the upper, middle, and lower portions of the left and right lungs to calculate the total score for each field of view at 200× (high magnification). The total pathological scores for lung injury were the sum of the four individual scores $(0-3$ for each), which were calculated from $6 \times 2$ fields of view by two pathology specialists and averaged to produce the final scores.

\section{Lung Wet-to-Dry (W/D) Weight Ratio}

Lungs were excised twenty-four hours after CLP surgery. Each lung was blotted dry to remove residual blood, weighed, and then placed in an oven at $70^{\circ} \mathrm{C}$ for $48 \mathrm{~h}$ to obtain the dry weight. The weight ratio of the wet lung to the dry lung was calculated to assess tissue edema.

ELISA

Mouse IL-1 $\beta$, IL-6, TNF- $\alpha$ and MPO levels in BALF were measured by ELISA. In brief, BALF was collected, and ELISA was performed according to the manufacturer's protocol (R\&D Systems Inc.).

\section{TUNEL Assay}

An In Situ Cell Death Detection Kit (Roche Diagnostics) was used to assess endothelial apoptosis according to the manufacturer's protocol. Lung tissues were fixed in $4 \%$ paraformaldehyde, embedded in paraffin, and digested with proteinase $\mathrm{K}$ for $20 \mathrm{~min}$. The sections were then incubated with the TUNEL reaction mixture $(50 \mu \mathrm{l})$ at $37^{\circ} \mathrm{C}$ for $1 \mathrm{~h}$. After three washes, a drop of freshly prepared diaminobenzidine solution was added to each slide; the slide was then incubated for $15 \mathrm{~min}$ and washed three times for 5 min each. The slides were stained with hematoxylin and hydrochloride alcohol, dehydrated in a graded series of alcohol, dried in xylene, and mounted with neutral gum. The pulmonary apoptosis index (AI) was determined by counting the number of apoptotic cells via light microscopy (400x). 


\section{Cellular Physiology Cell Physiol Biochem 2017;42:156-168

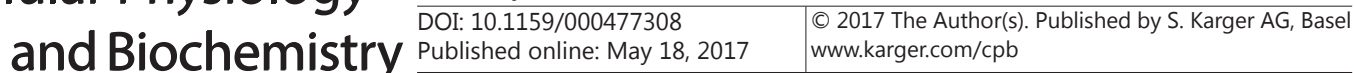

Chen et al.: HSPA12B Protects Vascular Endothelium in Sepsis-Induced ALI

Immunofluorescence staining

Lung tissues were removed and fixed with $4 \%$ paraformaldehyde, embedded in paraffin, and sectioned at $5 \mu \mathrm{m}$ for immunofluorescence staining. The tissue sections were incubated with PBS containing $1 \%$ bovine serum albumin, $0.1 \%$ Triton X-100 (PBS-BT), and 5\% normal serum for $1 \mathrm{~h}$ at room temperature. The solution was then removed and replaced with PBS containing anti-CD31 mAb (1:20) and anti-caspase-3 polyclonal antibody (1:100). After incubation for $2 \mathrm{~h}$ at room temperature, each tissue section was rinsed for 15 min with PBS containing $0.1 \%$ Triton X-100 and further incubated for $1 \mathrm{~h}$ with Alexa Fluor 488 donkey anti-rabbit IgG $(\mathrm{H}+\mathrm{L})$ antibody and Alexa Fluor 546 goat anti-rat IgG $(\mathrm{H}+\mathrm{L})$ antibody (Invitrogen), both diluted to 1:500 in PBS. The slides were rinsed again and incubated for $5 \mathrm{~min}$ with PBS containing $0.1 \mu \mathrm{g} / \mathrm{ml}$ DAPI (Millipore) to stain cell nuclei. The sections were analyzed with an Axioplan 2 fluorescence microscope (Zeiss, equipped with $40 x / 0.75$ and $63 x / 1.4$ objective lenses and AxioVision acquisition software). The intensity of the staining was evaluated using a microscope equipped for immunofluorescence analysis.

\section{Statistical Analysis}

Results are presented as the mean \pm SD from at least three independent experiments. Statistical analysis was performed using ANOVA followed by the Bonferroni post hoc test for comparison among multiple groups. A significant difference was defined as $P<0.05$.

\section{Results}

HSPA12B siRNA specifically inhibits HSPA12B expression in mouse lung

To identify the role of HSPA12B in sepsis-mediated ALI, we sought to knock down HSPA12B expression in vivo via nasal inhalation of HSPA12B siRNA. We designed and synthesized the siRNA sequence against rodent HSPA12B. To achieve lung transfection after the nasal inhalation, we used a novel nano-polymer-encapsulated siRNA. At $24 \mathrm{~h}$ after transfection, lung, liver, heart and kidney tissues were excised, and HSPA12B mRNA expression was detected. We determined that the nasal inhalation of HSPA12B siRNA was specifically effective at inhibiting HSPA12B expression in mouse lung tissue, with no significant changes occurring in other tissues (Fig. 1A). We also confirmed that HSPA12B siRNA has an initial attenuation effect on HSPA12B protein production during the first 24 $\mathrm{h}$ after transfection. These results suggest that the HSPA12B siRNA successfully inhibited and obviously downregulated HSPA12B expression compared with the NC siRNA (Fig. 1B). Therefore, the use of nano-polymer-encapsulated siRNA was demonstrated to be successful for silencing the HSPA12B gene with great specificity and potency.

\section{HSPA12B improves CLP-induced ALI survival rate}

To further determine the protective effects of HSPA12B in sepsis-related ALI, we transfected mice with HSPA12B siRNA or NC siRNA via intranasal administration $24 \mathrm{~h}$ before ALI was induced by CLP. Survival post-surgery was monitored every $24 \mathrm{~h}$ for a total of seven days to determine the survival rate. We have previously demonstrated in vivo that HSPA12B significantly improves survival in CLP-induced ALI. As shown in Fig. 2, all mice treated with HSPA12B siRNA died within 6 days; mice treated with NC siRNA and mice that underwent CLP surgery had $40 \%$ and $33.3 \%$ survival rates, respectively. These results show that HSPA12B knockdown reduced the survival of mice challenged with CLP surgery. Thus, we conclude that HSPA12B beneficially affects survival in sepsis-induced ALI.

\section{HSPA12B knockdown induces pathological changes in CLP-induced ALI}

$\mathrm{H} \& \mathrm{E}$ staining was used to assess pathological changes and examine the severity of lung injury in mice challenged with CLP surgery after the inhalation of HSPA12B siRNA. As shown in Fig. 3A, the control group showed normal lung structures. However, the HSPA12B siRNA group showed obvious pathological changes in the lungs, including hemorrhage, alveolar congestion, thickening of the alveolar wall/hyaline membrane formations, polymorphonuclear (PMN) cell infiltration and aggregation in airspaces or vessel walls, 
Fig. 1. Expression of HSPA12B in different tissues in vivo after HSPA12B siRNA nasal inhalation. (A) mRNA expression of HSPA12B in different tissues (e.g., heart, lung, kidney, and liver) after nasal inhalation of HSPA12B siRNA analyzed by RT-qPCR. (B) Western blot results showing the change in HSPA12B protein level in different tissues after nasal inhalation of HSPA12B siRNA. GAPDH was used as an internal control. All data are expressed as the mean \pm SD of three independent experiments $(\mathrm{n}=8) .{ }^{*} P<0.05$ vs. the NC siRNA group.
A

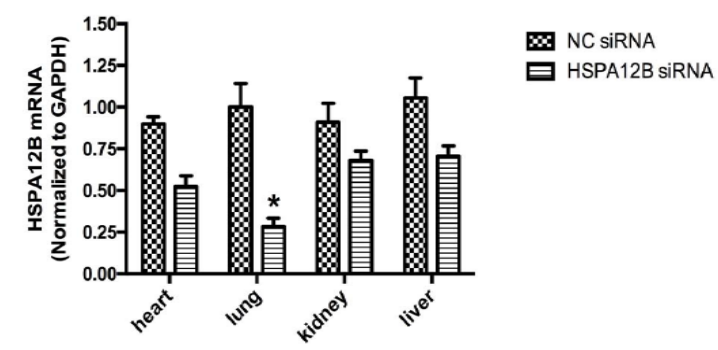

B
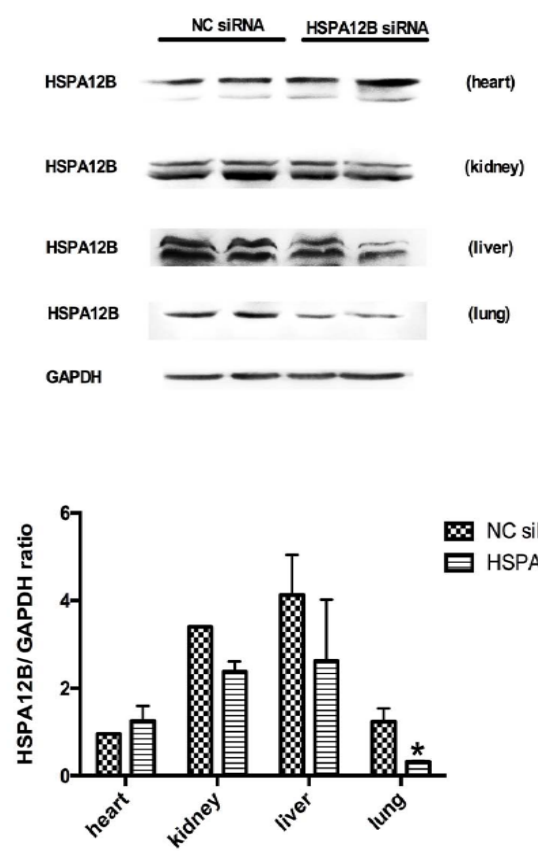

W NC siRNA HSPA12B SiRNA
Fig. 2. Survival curve after CLP surgery and nasal inhalation of HSPA12B siRNA. C57BL/6 mice $(\mathrm{n}=15$ per group) were pre-treated with HSPA12B SiRNA or NC siRNA before CLP surgery. Survival data were recorded every $24 \mathrm{~h}$ for 7 days. ${ }^{*} P<0.05$ vs. the NC siRNA group, ${ }^{\#} P<0.05$ vs. the sham group.

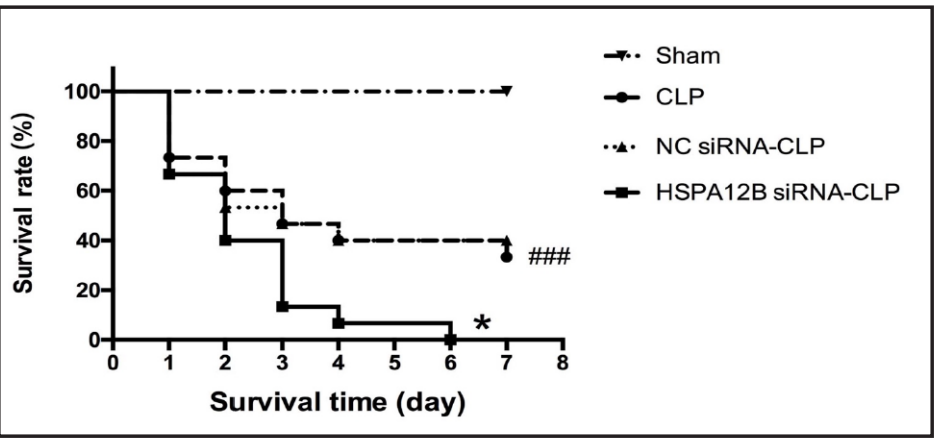

and edema. However, the CLP-induced pathological changes in lung tissues were attenuated in the NC siRNA and CLP group. Moreover, histological analysis showed that, compared with the mice that received CLP surgery after $48 \mathrm{~h}$, the mice in the HSPA12B siRNA group that received CLP surgery after $24 \mathrm{~h}$ had substantially more inflammatory cell infiltration, edema, hemorrhage, and alveolar wall thickening, as well as lower lung injury scores (Fig. 3B). Furthermore, the pathological scores in the HSPA12B siRNA group were significantly higher than those in the NC siRNA and CLP groups (Fig. 3C). Thus, knockdown of HSPA12B expression may accentuate lung tissue injury in CLP mice. 
Fig. 3. Histopathological analysis of lung tissues in mice challenged with CLP surgery after inhalation of HSPA12B siRNA. Lungs of mice pre-treated with HSPA12B SiRNA or NC SiRNA were obtained $24 \mathrm{~h}$ (A) or 48 h (B) after CLP surgery and embedded in paraffin. The embedded lungs were sectioned, and the sections were stained with H\&E. (C) Pathological lung injury scores. The data are representative examples of three individual lung sections.
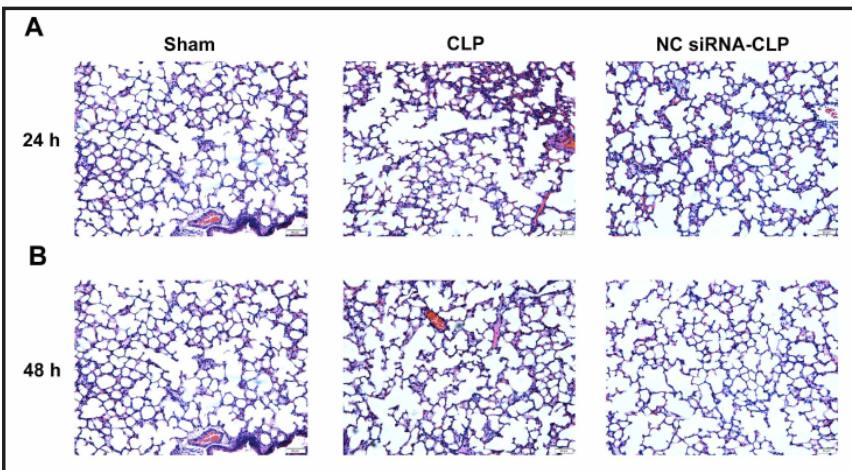
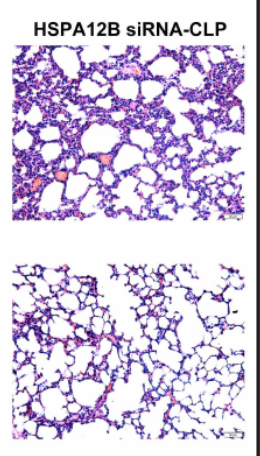

C
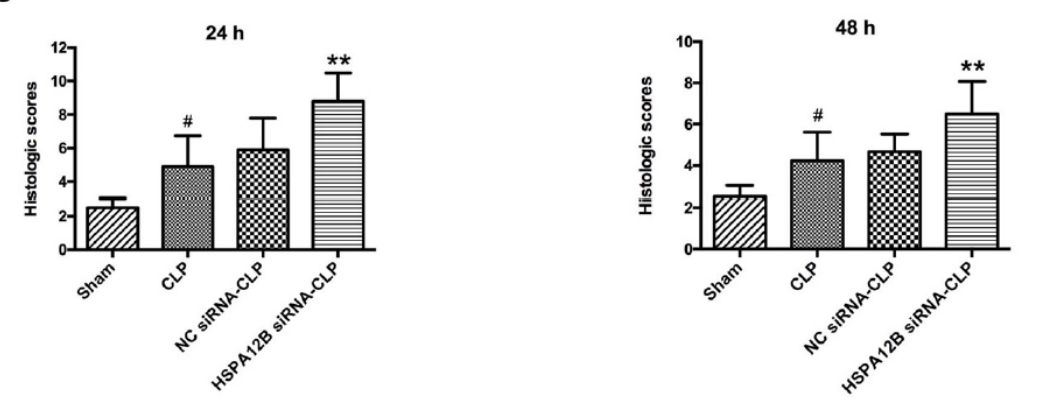

The scale bars in all images represent $50 \mu \mathrm{m}$. All data are expressed as the mean \pm SD of three independent experiments $(\mathrm{n}=8) .{ }^{*} P<0.05$ vs. the NC siRNA group, ${ }^{\#} P<0.05$ vs. the sham group.

HSPA12B siRNA increases inflammatory responses and neutrophil infiltration in sepsisinduced $A L I$

To investigate the effects of HSPA12B on the production of inflammatory mediators that are mechanistically linked to severe sepsis-induced lung injury, we assessed the levels of numerous inflammatory mediators that contribute to the pathogenesis of ALI. Our results showed that the levels of TNF- $\alpha$, IL- $1 \beta$, and IL- 6 in BALF were markedly increased in the HSPA12B siRNA group compared with the NC siRNA and CLP groups at $24 \mathrm{~h}$ after CLP surgery (Fig. 4A-C).

To further verify our findings, we examined the effect of HSPA12B in sepsis-induced ALI. The numbers of PMN cells in BALF were counted using flow cytometry; the results in the HSPA12B siRNA group were significantly different from those in the NC group at $24 \mathrm{~h}$ after CLP surgery (Fig. 4D). The percentage of PMN cells in BALF was significantly higher in the HSPA12B siRNA group than in the NC group. As expected, the lung water content (as measured by W/D weight ratio) was significantly higher in the HSPA12B siRNA group than in the NC and CLP groups (Fig. 4E). Analysis of BALF protein content was employed as an index of lung vascular permeability. As shown in Figure 4F, sepsis induced significant disruptions in lung vascular barrier function when compared to the sham group. Treatment of CLP mice with HSPA12B siRNA produced significant increase in BALF protein content when compared to the NC siRNA group. Moreover, there was no significance difference in the lung W/D weight ratio between the CLP and NC groups. The MPO activity results were consistent with those of the W/D weight ratio (Fig. 4G). MPO activity was low in the control sham-operation group. Exposure to the CLP challenge resulted in a significant increase in lung MPO activity, and the HSPA12B siRNA treatment significantly increased MPO activity. Thus, HSPA12B may ameliorate neutrophil infiltration and vascular leakage in septic lungs. Taken together, our data suggest that HSPA12B ameliorates CLP-induced lung injury and inflammatory responses.

\section{KARGER}




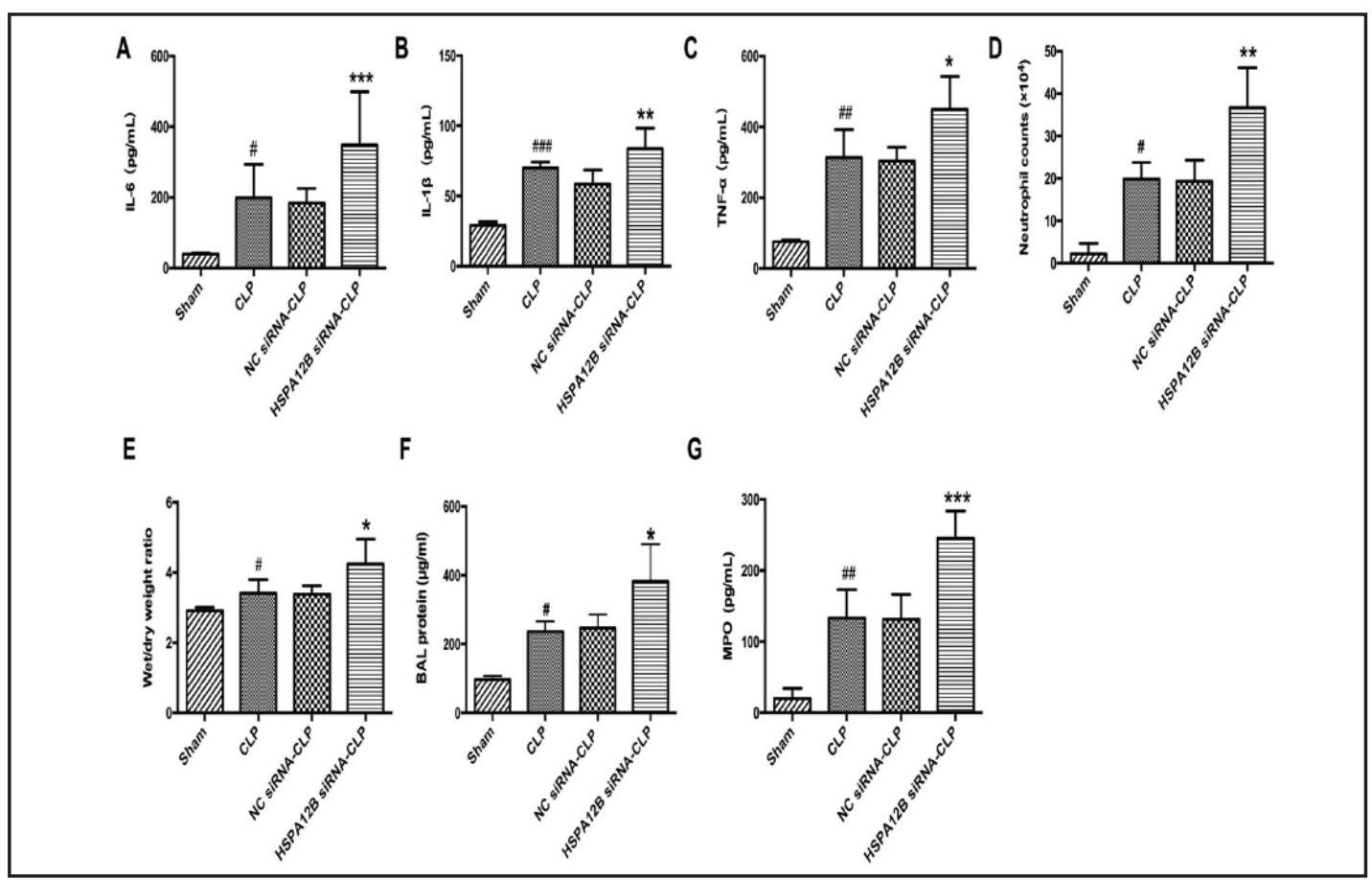

Fig. 4. Inflammatory responses and neutrophil infiltration in mice challenged with CLP surgery after inhalation of HSPA12B siRNA. Levels of IL-6 (A), IL-1 $\beta$ (B) and TNF- $\alpha$ (C) inflammatory cytokines in BALF were determined by ELISA. (D) Neutrophils (CD11 b+Gr-1 Ly6C $\left.\mathrm{C}^{+}\right)$in BALF were counted by flow cytometry. (E) The effects of HSPA12B on MPO activity in BALF were detected by ELISA. (F) The effects of HSPA12B on pulmonary edema were assessed by W/D weight ratios. All data are expressed as the mean \pm SD of three independent experiments $(\mathrm{n}=8)$. ${ }^{*} P<0.05$ vs. the NC siRNA group, ${ }^{*} P<0.05$ vs. the sham group.

HSPA12B protects the lungs against VE apoptosis via the inhibition of caspase-3 activation

To investigate whether HSPA12B could reduce VE apoptosis and thus protect the lungs, we next measured the level of apoptosis in the lung tissues using a TUNEL assay. No or few apoptotic cells were observed in lung tissue sections of the sham group, whereas more apoptotic cells were observed in the sections of the CLP and NC siRNA groups. Compared to the NC siRNA group, substantially more apoptotic cells were observed in the HSPA12B siRNA group (Fig. 5A), and the AI in the HSPA12B siRNA group was significantly higher than that in the NC siRNA group $(P<0.05)$ (Fig. 5B). To clarify whether the apoptosis of pulmonary endothelial cells could involve HSPA12B, lung tissue sections were double-stained with antiCD31 (red) and anti-cleaved caspase-3 (green). The immunofluorescence results showed that cleaved caspase- 3 was seldom expressed in the sham group; however, the number of CD31 and cleaved caspase-3 double-positive cells in the HSPA12B siRNA group was much greater than that in the NC siRNA group (Fig. 5C). Importantly, HSPA12B significantly reduced the expression of cleaved caspase-3 in lung tissues (Fig. 5D). These results indicate that HSPA12B reduced caspase- 3 activation and contributed to the reduction of sepsisinduced lung endothelial apoptosis.

HSPA12B protects the lungs against VE injury via the inhibition of ERK phosphorylation

To further identify the anti-inflammatory mechanism of HSPA12B, we explored the effects of HSPA12B on LPS-induced MAPK signaling, which is a major signaling pathway that plays essential roles in the production of pro-inflammatory cytokines and mediates the response to LPS. We examined phosphorylated-MAPK (i.e., JNK, ERK, and p38 MAPK) protein expression levels in lung tissues after CLP surgery. As shown in Fig. 6A, the level of p-ERK expression was significantly increased in the HSPA12B siRNA group. Furthermore, we detected the phosphorylated-MAPK (i.e., JNK, ERK, and p38 MAPK) protein expression levels 


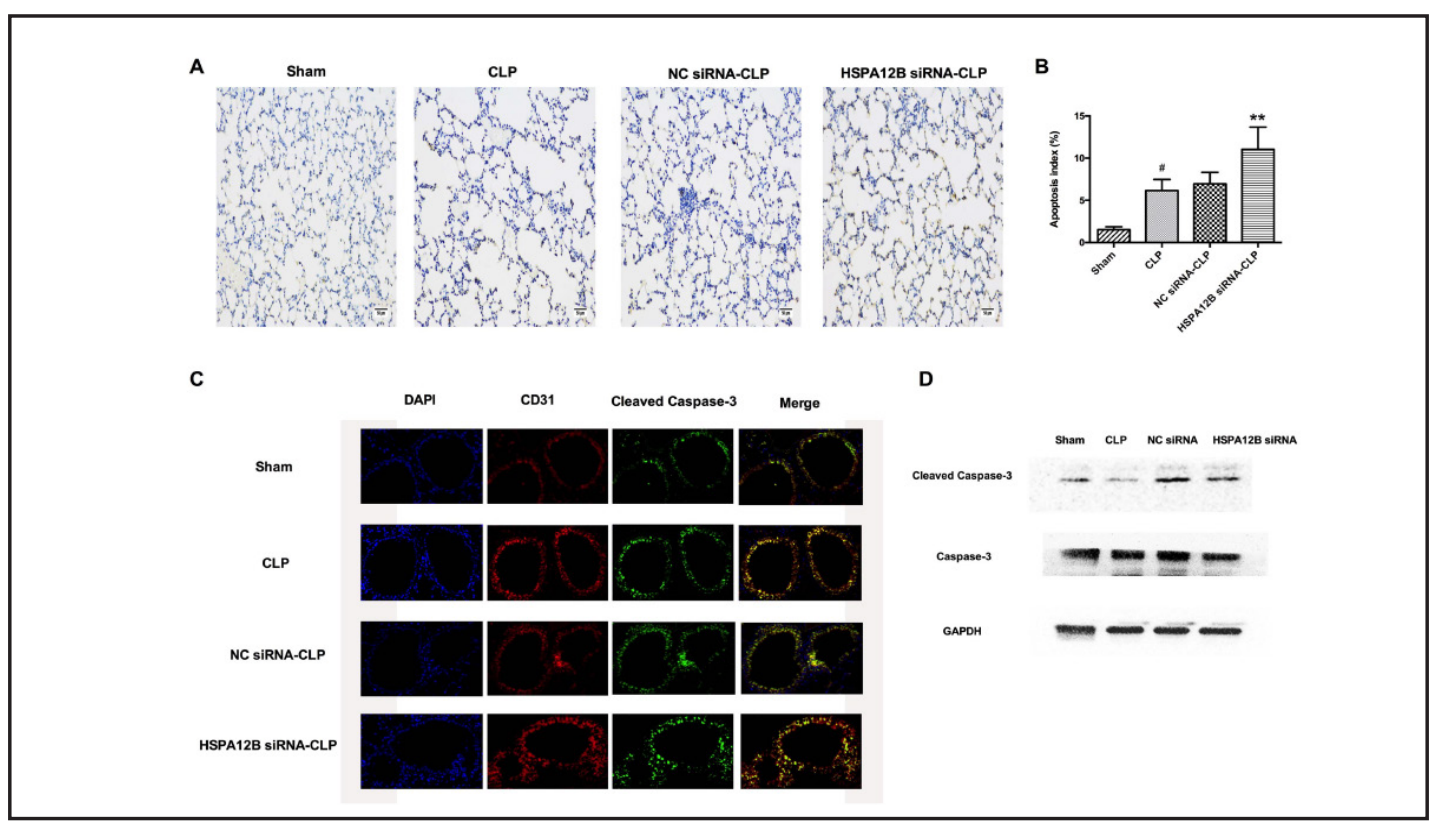

Fig. 5. Effects of HSPA12B knockdown by siRNA on endothelial cell apoptosis in vivo and in vitro. In vivo, lung tissues were harvested $24 \mathrm{~h}$ after CLP surgery from mice pre-treated with HSPA12B siRNA or NC siRNA. In vitro, HUVECs were pre-treated with HSPA12B plasmid or siRNA $12 \mathrm{~h}$ after LPS (1 $\mu \mathrm{g} / \mathrm{ml})$ stimulation. (A) Apoptosis in the lungs was assessed by a TUNEL assay. The arrows indicate cells positively stained for apoptosis. (B) The effects of HSPA12B on endothelial apoptosis were assessed by AI. (C) Expression of cleaved caspase-3 and CD31 in pulmonary vascular endothelium was determined by staining with both CD31 (red) and cleaved caspase-3 (green). (D) Protein expression of cleaved caspase-3 and caspase-3 in lung tissues was measured by Western blotting. The scale bars in all images represent $50 \mu \mathrm{m}$. GAPDH was used as an internal control. All data are expressed as the mean \pm SD of three independent experiments $(n=8)$. ${ }^{*} P<0.05$ vs. the NC siRNA group, ${ }^{\#} P<0.05$ vs. the sham group.

in HUVECs after LPS stimulation. The results showed that p-ERK expression was inhibited in the HSPA12B plasmid transfection group (Fig. 6B). These results indicate that HSPA12B protects the lungs against VE injury by inhibiting ERK phosphorylation.

\section{Discussion}

Here, we performed HSPA12B knockdown by using highly specific nano-polymerencapsulated siRNA to demonstrate that HSPA12B has a significant protective effect against pulmonary VE injury during sepsis-induced ALI. HSPA12B knockdown via siRNA transfection augments sepsis-induced endothelial injury and may potentially have therapeutic value in sepsis-induced ALI, for which effective interventions do not currently exist.

Pulmonary microvascular endothelial injury is critical to the pathogenesis of ALI during sepsis. Increased pulmonary microvascular permeability leads to the exudation of alveolar protein-rich liquid and subsequently to pulmonary edema and hyaline membrane formation. Pulmonary microvascular endothelial cells are important in the cellular mechanism of ALI pathogenesis. They attach to the lining of the vessel wall and regulate blood flow, thus facilitating the delivery of cells and proteins to tissue $[19,20]$. Difficulties in healing from endothelial cell injury can lead to the destruction of the blood-air barrier, which directly results in the leakage of lung tissue protein, thus initiating ALI and potentially severe ARDS $[21,22]$. The enhancement of endothelial barrier integrity is a novel treatment strategy for ALI/ARDS [23-26]. The identification of genes that modulate sepsis-induced endothelial dysfunction could potentially be relevant to a variety of organs.

\section{KARGER}




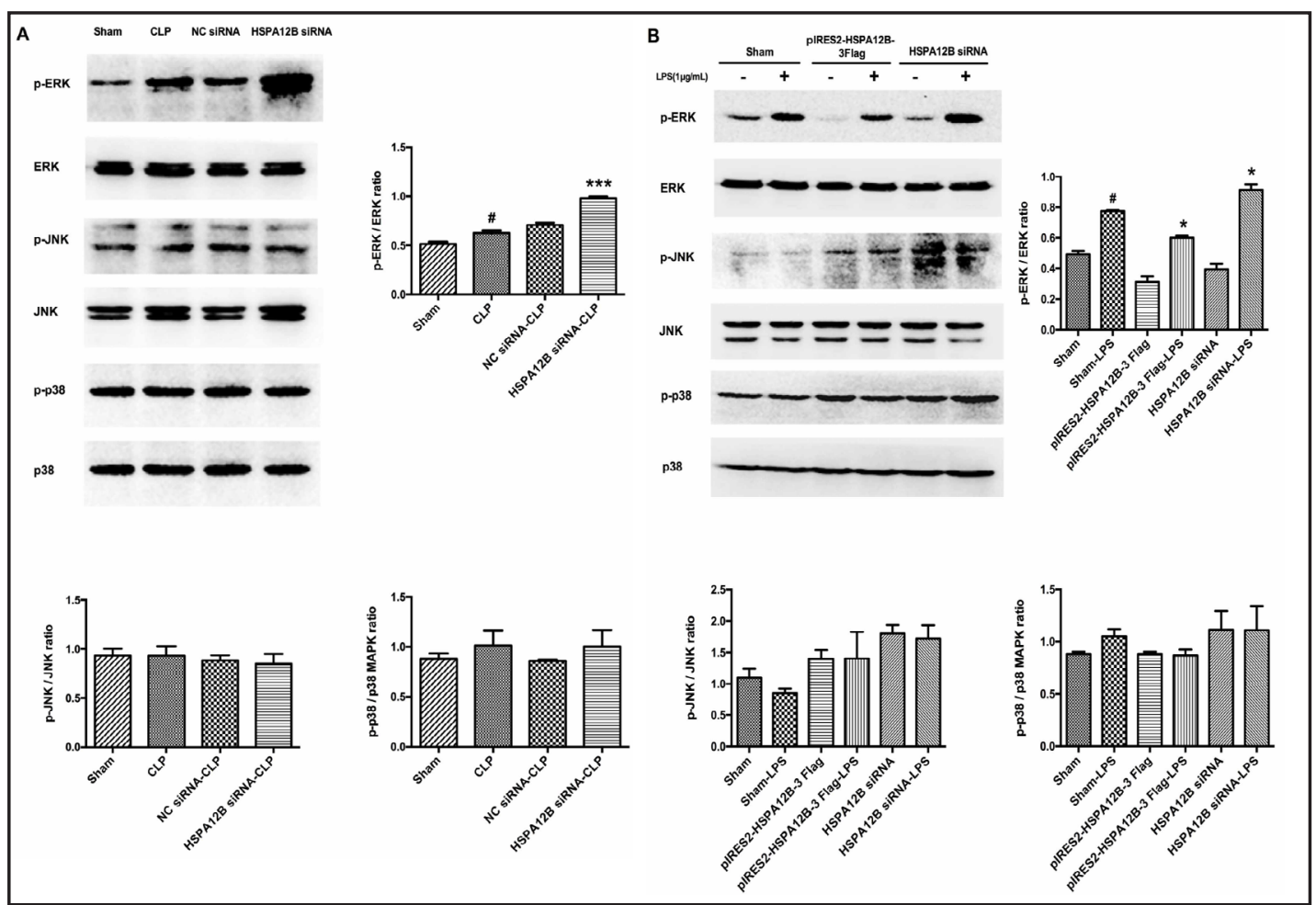

Fig. 6. Effects of HSPA12B on ERK/MAPK signaling in vivo and in vitro. Lung tissues were harvested 24 $\mathrm{h}$ after CLP surgery from mice pre-treated with HSPA12B siRNA or NC siRNA. HUVECs were pre-treated with HSPA12B plasmid or siRNA $12 \mathrm{~h}$ after LPS $(1 \mu \mathrm{g} / \mathrm{ml})$ stimulation. Protein expression levels of phosphorylated p38 MAPK, JNK and ERK in lung tissues (A) and HUVECs (B) were determined using Western blotting. The protein bands on the blots were analyzed using a densitometer, and the quantitative ratios for all samples were normalized to the corresponding total protein. All data are expressed as the mean \pm SD of three independent experiments $(n=8)$.

We observed a significantly higher mortality rate in the HSPA12B siRNA group than in the NC siRNA group, suggesting that knockdown of HSPA12B expression decreased the survival of mice with ALI after CLP surgery. Our previous studies have demonstrated that HSPA12B protects against sepsis-induced impairment in VE permeability by upregulating VE-cadherin. Collectively, our results suggest that nasal inhalation of HSPA12B siRNA aggravated sepsis-induced lung endothelial injury. Our findings also provide the first evidence of HSPA12B protection from sepsis-induced ALI.

Intypical ALI, lesions exhibit widespread destruction of alveolar epithelium and increased lung vascular permeability, alveolar hemorrhage, PMN cell infiltration, and severe histological damage $[27,28]$. Our studies showed that HSPA12B siRNA worsened the histopathological consequences of ALI, including hemorrhage, edema, alveolar wall thickness and PMN cell recruitment in mouse lungs after CLP. In ALI, neutrophils are important contributors to the progression of a host's inflammation defense $[29,30]$. Neutrophils migrate into the alveoli and release pro-inflammatory cytokines, further damaging the pulmonary microvascular endothelial cells [31,32]. Herein, we have shown that the number of PMN cells was higher in the HSPA12B siRNA group than in the NC siRNA group. These findings suggest that HSPA12B knockdown leads to heightened inflammatory responses in the lungs. Pro-inflammatory cytokines, such as TNF- $\alpha$, IL- $1 \beta$, and IL- 6 , are considered pivotal mediators for initiating, amplifying, and perpetuating sepsis-induced lung injury [33-35]. These cytokines mediate phagocytic cell adhesion, vascular dysfunction, leukocyte recruitment, and tissue damage, as well as potentiate death during sepsis [36]. To determine whether these mediators are associated with the protective effect of HSPA12B against sepsis-induced lung endothelial

\section{KARGER}




\section{Cellular Physiology Cell Physiol Biochem 2017;42:156-168 \begin{tabular}{l|l} 
DOI: 10.1159/000477308 & Ond 2017 The Author(s). Published by S. Karger AG, Basel \\
www.karger.com/cpb
\end{tabular} \\ Chen et al.: HSPA12B Protects Vascular Endothelium in Sepsis-Induced ALI}

injury, we examined the levels of TNF- $\alpha$, IL-1 $\beta$, and IL- 6 in BALF. The results showed that HSPA12B downregulation enhanced the levels of TNF- $\alpha$, IL-1 $\beta$, and IL- 6 in septic mice. MPO is highly expressed in neutrophils and hemoglobin. When neutrophils are activated, MPO can be released into the phagosomes and the extracellular matrix. As such, the immune activity of MPO may reflect the extent of vascular injury [37]. The results showed that HSPA12B could reduce MPO activity. Additionally, we confirmed previous findings that HSPA12B protects pulmonary VE cell permeability and reduces pulmonary edema in sepsis. Collectively, these data suggest that HSPA12B knockdown may aggravate inflammatory responses and pathological injury in sepsis-induced ALI.

There is an increasing amount of evidence implicating increased epithelial/endothelial cell apoptosis in the pathogenesis of ALI. Studies in critically ill patients have shown that ALI is associated with increased cell death [38-40]. Our results showed that HSPA12B siRNA could increase AI in mouse lungs. Caspase- 3 plays a key role in apoptotic cell death [41, 42], and our analysis revealed a significant increase in the level of cleaved caspase-3 after CLP surgery, indicating increased cell apoptosis in the lungs. Immunofluorescence staining was performed to verify the apoptosis of pulmonary endothelial cells induced by CLP. As shown, lung tissues co-stained with antibodies against CD31 and cleaved caspase- 3 appeared orange, indicating apoptosis. In accordance with our hypothesis, endothelial apoptosis increased after HSPA12B knockdown, and caspase-3 activation was involved in this apoptotic process.

MAPK family molecules are important regulators involved in the production of cytokines and mediators associated with the pathogenesis of inflammatory processes [43, 44]. HSPA12B could be a key upstream component of MAPK activation [45]. In addition, some studies have reported that HSPA12B expression is mediated by activation of the JNK and p38 signaling pathways, but not the ERK1/2 MAPK signaling pathway, upon LPS-induced astrocyte activation. However, our research showed that HSPA12B protects the lungs against VE injury by inhibiting ERK phosphorylation.

A limitation of our study is that it focused on the first 24 hours after CLP, and the question of whether HSPA12B exerts similar beneficial effects at other times remains to be investigated. As severe sepsis occurred at 24 hours after CLP, we chose this time point to assess ALI. Another shortcoming of our study is that HUVECs may not perfectly represent the expression of HSPA12B in the lungs. Therefore, further research should be performed to validate whether pulmonary microvascular cells participate in the observed effects of HSPA12B in sepsis-induced ALI.

In summary, HSPA12B knockdown significantly aggravated CLP-induced lung injury and significantly decreased the survival rate of septic mice. Inhalation of HSPA12B siRNA in CLPinduced ALI led to increased lung W/D weight ratio, inflammatory PMN cell infiltration into lung tissue, and inflammatory mediator release, which exacerbated both lung pathological features and pulmonary edema. Additionally, HSPA12B reduced lung endothelial apoptosis, protecting against sepsis-induced ALI. The beneficial effect of HSPA12B against lung endothelial injury also involved inhibition of the ERK/MAPK pathway and caspase-3 activation. Although more studies are needed to fully clarify the potential role of HSPA12B in sepsis-induced ALI and the pathogenic events that lead to the development of multiple organ failure, our results suggest that HSPA12B is a potentially useful target that provides a new strategy for the clinical prevention and treatment of ALI/ARDS in sepsis.

\section{Acknowledgements}

This study was supported by the National Natural Science Foundation of China (NO. 81270128) to Keming Zhu.

\section{Disclosure Statement}

The authors declare that there is no conflict of interest regarding publication of this manuscript. 


\section{Cellular Physiology Cell Physiol Biochem 2017;42:156-168

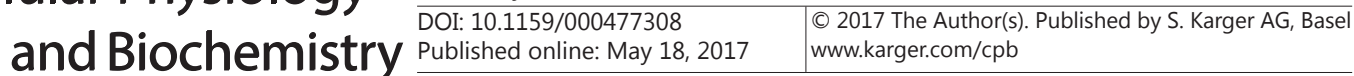

Chen et al.: HSPA12B Protects Vascular Endothelium in Sepsis-Induced ALI

\section{References}

1 Cohen J: The immunopathogenesis of sepsis. Nature 2002;420:885-891.

-2 ARDS Definition Task Force: Acute respiratory distress syndrome. JAMA 2012;307:2526-2533.

-3 Matthay MA, Ware LB, Zimmerman GA: The acute respiratory distress syndrome. J Clin Invest 2012;122:2731-2740.

-4 Hernu R, Wallet F, Thiollière F, Martin O, Richard JC, Schmitt Z, Wallon G, Delannoy B, Rimmelé T, Démaret C, Magnin C, Vallin H, Lepape A, Baboi L, Argaud L, Piriou V, Allaouchiche B, Aubrun F, Bastien O, Lehot JJ, Ayzac L, Guérin C: An attempt to validate the modification of the AmericanEuropean consensus definition of acute lung injury/acute respiratory distress syndrome by the Berlin definition in a university hospital. Intensive Care Med 2013;39:2161-2170.

-5 Herold S, Gabrielli NM, Vadász I: Novel concepts of acute lung injury and alveolar-capillary barrier dysfunction. Am J Physiol Lung Cell Mol Physiol 2013;305:L665-L681.

-6 Matute-Bello G, Downey G, Moore BB, Groshong SD, Matthay MA, Slutsky AS, Kuebler WM, Acute Lung Injury in Animals Study Group: An official American Thoracic Society workshop report: features and measurements of experimental acute lung injury in animals. Am J Respir Cell Mol Biol 2011;44:725-738.

7 Ince C, Mayeux PR, Nguyen T, Gomez H, Kellum JA, Ospina-Tascón GA, Hernandez G, Murray P, De Backer D, ADQI XIV Workgroup: The endothelium in sepsis. Shock 2016;45:259-270.

8 Perl M, Lomas-Neira J, Venet F, Chung CS, Ayala A: Pathogenesis of indirect (secondary) acute lung injury. Expert Rev Respir Med 2011;5:115-126.

-9 Zouein FA, Kurdi M, Booz GW:HSPA12B and repairing the heart: beauty in simplicity. Cardiovasc Res 2013;99:587-589.

10 Shi J, Yang D, Cong X, Li Y, Yang X, Liu Y: Expression of HSPA12B in acute cardiac allograft rejection in rats. Pathol Res Pract 2015;211:20-26.

11 Kang L, Zhang G, Yan Y, Ke K, Wu X, Gao Y, Li J, Zhu L, Wu Q, Zhou Z: The role of HSPA12B in regulating neuronal apoptosis. Neurochem Res 2013;38:311-320.

12 Zhou H, Qian J, Li C, Li J, Zhang X, Ding Z, Gao X, Han Z, Cheng Y, Liu L: Attenuation of cardiac dysfunction by HSPA12B in endotoxin-induced sepsis in mice through a PI3K-dependent mechanism. Cardiovasc Res 2011;89:109-118.

-13 Li J, Zhang Y, Li C, Xie J, Liu Y, Zhu W, Zhang X, Jiang S, Liu L, Ding Z:HSPA12B attenuates cardiac dysfunction and remodelling after myocardial infarction through an eNOS-dependent mechanism. Cardiovasc Res 2013;99:674-684.

14 Wu J, Li X, Huang L, Jiang S, Tu F, Zhang X, Ma H, Li R, Li C, Li Y, Ding Z, Liu L: HSPA12B inhibits lipopolysaccharide-induced inflammatory response in human umbilical vein endothelial cells. J Cell Mol Med 2015;19:544-554.

-15 Kang Q, Chen Y, Zhang X, Yu G, Wan X, Wang J, Bo L, Zhu K: Heat shock protein A12B protects against sepsis-induced impairment in vascular endothelial permeability. J Surg Res 2016;202:87-94.

-16 Zhang R, Wan XJ, Zhang X, Kang QX, Bian JJ, Yu GF, Zhu KM, HSPA P:Plasma HSPA12B is a potential predictor for poor outcome in severe sepsis. PLOS ONE 2014;9:e101215.

17 Rittirsch D, Huber-Lang MS, Flierl MA, Ward PA: Immunodesign of experimental sepsis by cecal ligation and puncture. Nat Protoc 2009;4:31-36.

-18 Carraway MS, Welty-Wolf KE, Miller DL, Ortel TL, Idell S, Ghio AJ, Petersen LC, Piantadosi CA: Blockade of tissue factor:treatment for organ injury in established sepsis. Am J Respir Crit Care Med 2003;167:1200-1209.

19 Matthay MA, Zimmerman GA: Acute lung injury and the acute respiratory distress syndrome: four decades of inquiry into pathogenesis and rational management. Am J Respir Cell Mol Biol 2005;33:319-327.

20 Orfanos SE, Mavrommati I, Korovesi I, Roussos C: Pulmonary endothelium in acute lung injury: from basic science to the critically ill. Intensive Care Med 2004;30:1702-1714.

21 Rubenfeld GD, Caldwell E, Peabody E, Weaver J, Martin DP, Neff M, Stern EJ,Hudson LD: Incidence and outcomes of acute acute lung injury. N Engl J Med 2005;353:1685 -1693.

22 Goldenberg NM, Steinberg BE, Slutsky AS, Lee WL: Broken barriers: a new take on sepsis pathogenesis. Sci Transl Med 2011;3:88ps25. 


\section{Cellular Physiology Cell Physiol Biochem 2017;42:156-168 \begin{tabular}{l|l} 
DOI: 10.1159/000477308 & and Biochemistry \\
Published online: Nay 18, 2017 & $\begin{array}{l}\text { O 2017 The Author(s). Published by S. Karger AG, Basel } \\
\text { www.karger.com/cpb }\end{array}$
\end{tabular}}

Chen et al.: HSPA12B Protects Vascular Endothelium in Sepsis-Induced ALI

23 Minamino T, Komuro I: Regeneration of the endothelium as a novel therapeutic strategy for acute acute lung injury. J Clin Invest 2006;116:2316-2319.

24 Bitko V, Barik S: Respiratory viral diseases: access to RNA interference therapy. Drug Discov Today Ther Strateg 2007;4:273-276.

-25 Herold S, Gabrielli NM, Vadász I: Novel concepts of acute lung injury and alveolar-capillary barrier dysfunction. Am J Physiol Lung Cell Mol Physiol 2013;305:665-681.

-26 Bhattacharya J, Matthay MA: Regulation and repair of the alveolar-capillary barrier in acute lung injury. Annu Rev Physiol 2013;75:593-615.

-27 Abraham E, Singer M: Mechanisms of sepsis-induced organ dysfunction. Crit Care Med 2007;35:2408-2416.

-28 Matthay MA, Zemans RL: The acute respiratory distress syndrome: pathogenesis and treatment. Annu Rev Pathol 2011;6:147e163:147-163.

-29 Brown KA, Brain SD, Pearson JD, Edgeworth JD, Lewis SM, Treacher DF: Neutrophils in development of multiple organ failure in sepsis. Lancet 2006;368:157-169.

- 30 Yang KY, Arcaroli JJ, Abraham E: Early alterations in neutrophil activation are associated with outcome in acute lung injury. Am J Respir Crit Care Med 2003;167: 1567-1574.

-31 Zhang HX, Liu SJ, Tang XL, Duan GL, Ni X, Zhu XY, Liu YJ, Wang CN. H2SAttenuates LPS-Induced Acute Lung Injury by Reducing Oxidative/Nitrative Stressand Inflammation. Cell Physiol Biochem 2016;40:1603-1612.

-32 Grammes J, Soehnlein O: Contribution of neutrophils to acute lung injury. Mol Med 2011;17:293-307.

-33 Bhatia M, Moochhala S: Role of inflammatory mediators in the pathophysiology of acute respiratory distress syndrome. J Pathol 2004;202:145-156.

-34 Zhu J, Duan G, Lang L, Liu Y, Zhu J, Wang H, Liu Y. The bacterial component flagellin induces antisepsis protection through TLR-5, IL-1RN and VCAN during polymicrobial sepsis in mice. Cell Physiol Biochem 2015; 36: 446-56.

- 35 Park WY, Goodman RB, Steinberg KP, Ruzinski JT, Radella F, Park DR, Pugin J, Skerrett SJ, Hudson LD, Martin TR: Cytokine balance in the lungs of patients with acute respiratory distress syndrome. Am J Respir Crit Care Med 2001;164:1896-1903.

- 36 Rittirsch D, Flierl MA, Ward PA: Harmful molecular mechanisms in sepsis. Nat Rev Immunol 2008;8:776-787.

-37 Lau D, Baldus S: Myeloperoxidase and its contributory role in inflammatory vascular disease. Pharmacol Ther 2006;111:16-26.

38 Martin TR, Nakamura M, Matute-Bello G: The role of apoptosis in acute lung injury. Crit Care Med 2003;31 (4 Suppl):S184-S188.

-39 Chopra M, Reuben JS, Sharma AC: Acute Lung Injury:Apoptosis and signaling mechanisms. Exp Biol Med 2009;234:361-371.

- 40 Perl M, Chung CS, Perl U, Thakkar R, Lomas-Neira J, Ayala A: Therapeutic accessibility of caspase mediated cell death as a key pathomechanism in indirect acute lung injury. Crit Care Med 2010;38:1179-1186.

41 Porter AG, Jänicke RU: Emerging roles of caspase-3 in apoptosis. Cell Death Differ 1999;6:99-104.

-42 Bröker LE, Kruyt FA, Giaccone G: Cell death independent of caspase: a review. Clin Cancer Res 2005;11:3155-3162.

43 Lee IT, Yang CM: Inflammatory signalings involved in airway and pulmonary diseases. Mediators Inflamm 2013;2013:791231.

-44 Hu R, Xu H, Jiang H, Zhang Y, Sun Y: The role of TLR4 in the pathogenesis of indirect acute lung injury. Front Biosci 2013;18:1244-1255.

-45 Li XH, Huang J, Yuan DM, Cheng C, Shen AG, Zhang DM, Tao T, Liu YH, Lu JJ, Guo YB, Zhu H, Chen J, Lu $\mathrm{X}$ : HSPA12B regulates SSeCKS-mediated astrocyte inflammatory activation in neuroinflammation. Exp Cell Res 2015;339:310-319. 